\title{
DESENVOLVIMENTO DE UM SMART GATEWAY PARA CONSTRUÇÃO CIVIL UTILIZANDO BIM, IOT E RFID
}

\author{
Caroline Araújo $^{(1)}$, Caio Mendes ${ }^{(1)}$, Emerson Ferreira ${ }^{(1)}$, Dayana Costa ${ }^{(1)}$, Cassio \\ Serafim ${ }^{(1)}$, Julio Santos ${ }^{(1)}$ \\ (1) Universidade Federal da Bahia, Salvador, Brasil
}

\begin{abstract}
Resumo
A dificuldade de gerenciar os diferentes dados envolvidos nos processos produtivos da construção civil causa deficiências no fluxo de informações necessárias, o que contribui para um cenário de baixa produtividade. O desenvolvimento de tecnologias e sistemas interdisciplinares associados à Indústria 4.0 tem potencial para trazer melhorias para o setor. $\mathrm{O}$ BIM oferece suporte aos avanços tecnológicos e serve de interface natural para a implantação da IoT e da Computação em Nuvem na construção. Porém, alguns desafios ainda são observados na estruturação prática de sistemas que buscam integrar estas tecnologias. Além disso, são observadas dificuldades para garantir a disponibilidade de redes de comunicação em toda a área do canteiro. O objetivo deste estudo é desenvolver um gateway que desempenhe o papel de conectar elementos de um sistema RFID, de forma a se comunicar com redes internas e externas e atuar na transferência de dados em tempo real. A estratégia metodológica adotada é a pesquisa exploratória e experimental. Como resultado, obteve-se o gateway com capacidade de: 1) gerenciar os dados coletados; 2) selecionar e processar os dados relevantes; 3) armazenar localmente os dados e 4) servir de interface para agregar informações de interesse no banco de dados online para alimentar o modelo BIM, onde estas informações são contextualizadas. As principais aplicações previstas são no rastreamento de recursos físicos, planejamento e controle de produção e controle de qualidade.
\end{abstract}

\section{Introdução}

A construção civil ainda apresenta um baixo desempenho quando comparada à outras indústrias e uma das razões para que isso aconteça é o fornecimento inadequado das informações ao longo do desenvolvimento dos projetos e obras [4]. O cenário da quarta revolução industrial, conhecida como Indústria 4.0, indica que Tecnologias de Informação e Comunicação (TIC) interdisciplinares devem ser cada vez mais usadas para melhorar o desempenho do setor ao permitir a digitalização, automação e integração ao longo de toda a cadeia de valor da construção $[11,13]$. 
Oesterreich e Teuteberg [11] identificaram na literatura que a Modelagem da Informação da Construção (BIM), a Internet das Coisas (IoT) e a Computação em Nuvem são tecnologias centrais para a construção civil no contexto da Indústria 4.0, sendo que a palavra BIM foi a que apareceu com mais frequência nas publicações acadêmicas investigadas pelos referidos autores, confirmando o seu papel como uma tecnologia importante e consolidada na construção.

O BIM surgiu para melhorar os fluxos de informação na construção e se apresenta como uma plataforma digital para criar, trocar e gerenciar informações relevantes no contexto das construções [4]. O BIM tem beneficiado a construção civil devido a sua capacidade de produzir modelos virtuais cada vez mais precisos para representar a realidade, que facilitam a comunicação e colaboração entre proprietários, arquitetos, consultores, contratados, fabricantes e também operadores, promovendo o desenvolvimento de projetos mais integrados e eficientes [5].

A IoT, por sua vez, pode ser definida como um conjunto de artefatos associados a sistemas incorporados que podem conter mecanismos elétricos, mecânicos, computacionais e de comunicação para permitir a troca de dados via Internet [15]. Além de ser capaz de estabelecer comunicação entre todos os dispositivos reais ou virtuais que existem no cotidiano humano, concebendo ambientes inteligentes, a IoT também é capaz de detectar mudanças de status destes dispositivos em tempo real [8]. A Identificação por Rádio Frequência (RFID) é uma tecnologia de identificação de elementos cujos sistemas de leitura podem discernir diferentes tags localizadas em uma mesma área sem a necessidade de assistência humana [16]. O seu desenvolvimento serviu como base para constituir a IoT da forma como é conhecida atualmente [14].

O uso integrado destas tecnologias pode ser utilizado em métodos para rastreamento e gerenciamento de recursos físicos no canteiro de obras. Estes métodos são aplicados com o objetivo de fornecer mais controle sobre os processos construtivos e, consequentemente, trazer melhorias em termos de qualidade, produtividade e segurança para as obras [12]. Segundo Gao et al. [6], os modelos BIM oferecem potencial para serem utilizados como "gêmeos digitais" do ambiente construído representado, fornecendo um contexto espacial refinado para as informações coletadas com sistemas de IoT.

Porém, alguns desafios ainda são observados na estruturação prática de sistemas que buscam integrar as tecnologias BIM, IoT e RFID para aplicação na construção. Existem problemas relacionados, por exemplo, aos processos de seleção, destinação e armazenamento adequado de cada informação conforme a sua relevância. Segundo Atzori, Iera e Morabito [2], um sistema IoT para lidar com informações efetivamente relevantes envolve diferentes conteúdos e requer políticas de endereçamento eficazes, sendo recomendável a utilização de um gateway configurado entre a rede e o sistema RFID. Além disso, segundo Chen et al. [4], não é recomendado importar diretamente os dados brutos coletados no BIM, por isso um gateway inteligente pode ser utilizado para atuar como middleware (intermediário) entre a camada física do projeto e a camada BIM, garantindo a integração das informações entre as duas camadas.

Neste sentido, o objetivo do presente estudo é desenvolver um gateway que desempenhe o papel de estabelecer conexões entre diferentes dispositivos RFID, visando se comunicar com redes internas e externas e atuar na transferência de dados em tempo real para permitir a integração da IoT com o BIM. 


\section{Fundamentação Teórica}

Zhai et al. [17] desenvolveram uma plataforma BIM habilitada para IoT em sistemas préfabricados (Construção Modular Integrada) utilizando um Estudo de Caso como metodologia. O gateway, neste caso, tem as seguintes atribuições: 1) conecta um conjunto de objetos inteligentes (objetos associados a tags inteligentes, como as do sistema RFID) através de padrões de comunicação sem fio; 2) permite que os operadores acessem informações como o status dos objetos inteligentes para executar suas tarefas diárias; 3) atua como a ponte que se comunica com as camadas superiores, ou seja, Plataforma como um Serviço (PaaS) e Software como um Serviço (SaaS) e 4) é capaz de pré-processar e armazenar em cache dados em tempo real local e temporal, caso a rede de comunicação não esteja disponível, o que ajuda a eliminar o risco de perda de informações. Ao combinar a tecnologia Auto-ID com o gateway, a identificação exclusiva de todos os tipos de objetos de construção pode ser feita em todas as fases desde a montagem até a manutenção das peças.

Li et al. [9] desenvolveram um sistema que integra a IoT e o BIM para projetos de habitação pública com pré-fabricados em Hong Kong utilizando como metodologia a Design Science Research (DSR). Zhong et al. [18] desenvolveram uma plataforma multidimensional habilitada para IoT e BIM para obter visibilidade e rastreabilidade em tempo real na construção préfabricada utilizando um Estudo de Caso como metodologia. Ambos os referidos autores, Li et al. [9] e Zhong et al. [18], utilizam um gateway que: 1) conecta e hospeda um conjunto de objetos inteligentes através de padrões de comunicação com ou sem fio, além de permitir acesso ao status dos objetos inteligentes; 2) comunica e interage com os sistemas de tomada de decisão de nível superior, fornecendo informações úteis e em tempo real em formato padronizado; 3 ) processa, armazena em cache e troca dados e eventos em tempo real local e temporalmente e 4) fornece um rico conjunto de facilidades para definição, configuração e execução de serviços. Chen et al. [4] e Chen et al. [3] exploraram um gateway inteligente para uso em conjunto com o BIM e na construção utilizando como metodologia o Estudo Exploratório, onde o gateway era capaz de: 1) conectar e hospedar um conjunto de objetos habilitados para RFID; 2) comunicar e interagir com o sistema de apoio à decisão; 3) processar e trocar dados em tempo real e 4) fornecer recursos para definição e configuração de serviços.

Apesar das exemplificações a respeito do funcionamento do sistema em geral, os autores mencionados não abordaram de forma detalhada o processo de construção do gateway com requisitos técnicos e detalhes para uma possível replicação. Andrade et al. [1] desenvolveram e detalharam a plataforma SOFT-IoT, que serve de base para a criação de um gateway e cujos procedimentos adotados serviram de base para o presente estudo. SOFT-IoT é uma interface que introduz o conceito de Névoa das Coisas (FoT, do inglês Fog of Things) explorando o processamento, armazenamento e a capacidade de rede dos recursos locais, permitindo a integração de diferentes dispositivos em uma arquitetura IoT [1].

\section{Metodologia}

\subsection{Delineamento da Pesquisa}

A estratégia metodológica adotada é a pesquisa exploratória e experimental, que tem como objetivo principal o aprimoramento de ideias, possibilitando que diferentes aspectos relativos 
ao fato estudado sejam considerados $[7,10]$. As etapas que determinam o delineamento da pesquisa estão indicadas na Figura 1.

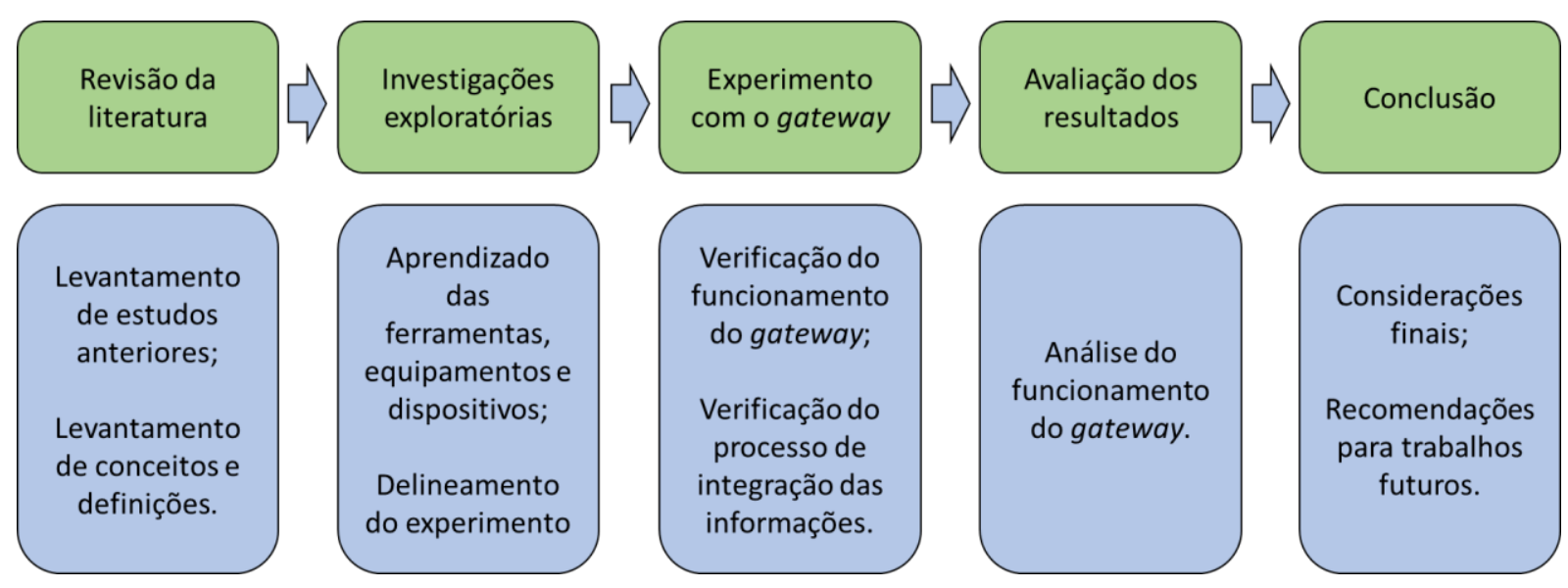

Figura 1: Etapas de pesquisa.

O estudo envolveu uma revisão da literatura para compreensão do tema, dos conceitos e das definições. Foram realizadas investigações exploratórias para aprendizado das ferramentas, equipamentos e dispositivos necessários. Em seguida foi desenvolvido e analisado o gateway, compreendendo todas as funcionalidades e capacidades necessária para o contexto no qual foi proposto. Por fim, foram feitas as considerações finais e recomendações de trabalhos futuros.

\subsection{Experimento}

A Figura 2 indica a estrutura geral adotada no experimento e onde o gateway será aplicado nesta estrutura. O Ambiente Físico do canteiro de obras, no qual o sistema RFID atua para rastrear recursos físicos da construção, deve se comunicar com o Ambiente Virtual proporcionado pelo BIM e pelo Website. As informações relevantes devem chegar ao usuário final através da visualização do Website, no qual estará disponível a respectiva versão online do modelo BIM. Para tanto, o gateway seve como middleware entre o Ambiente Físico e o Ambiente Virtual, atuando em conjunto com o banco de dados.

O experimento foi desenvolvido utilizando os dispositivos Raspberry PI Zero W e Raspberry PI 3, bem como as ferramentas Firebase (como banco de dados online), SQLite, Unity e Revit Autodesk. Os dados coletados são provenientes do sistema RFID composto por um leitor MFRC-522 e tags de baixa frequência embutidas em chaveiros, além de dispositivos auxiliares como LED's, buzzer, jumpers, protoboard, baterias e quatro push buttons que serviram para interação com o usuário. 


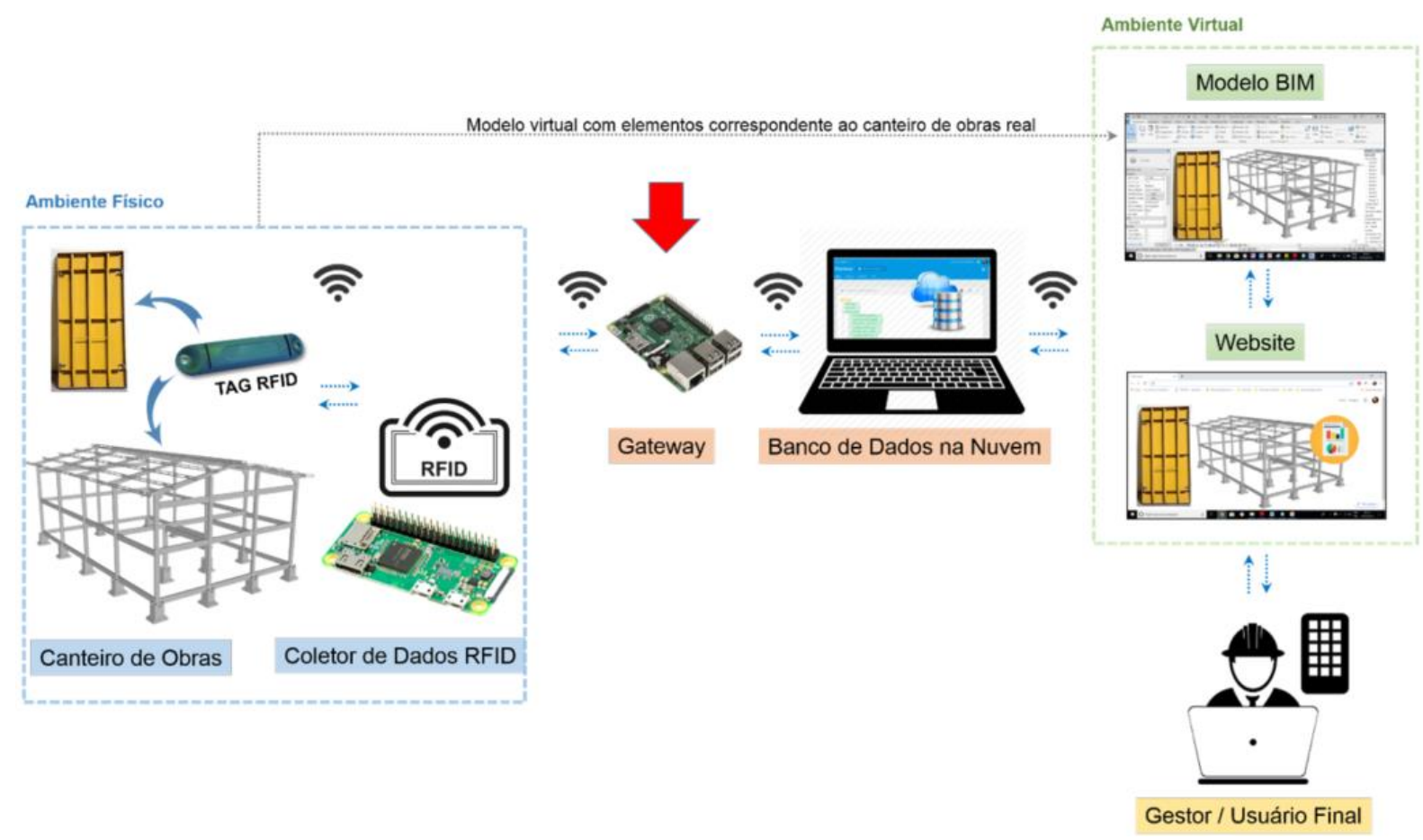

Figura 2: Estrutura geral do experimento.

\subsubsection{Procedimentos para Desenvolver o Gateway}

O desenvolvimento do gateway inteligente foi baseado na plataforma SOFT-IoT e utilizou-se o Raspberry PI 3. O procedimento inicial para permitir a aplicação desenvolvida no gateway consistiu em quatro etapas: (1) instalação do sistema operacional Raspbian; (2) instalação dos pacotes necessários para a aplicação em Python, (3) configuração do Raspberry PI 3 para tornálo um roteador de rede local e (4) configurações necessárias para que o gateway acesse tanto a sua própria rede Wi-Fi local e quanto a rede cabeada WLAN. Dessa forma, para a construção do servidor foi necessária a criação de uma pasta local no gateway para que todos os arquivos fossem alocados de forma correta e organizada. Após as configurações e testes referentes ao funcionamento de cada arquivo e já considerando a conexão com o sistema RFID, o Raspberry PI3 tornou-se um gateway capaz de lidar com os dados conforme a necessidade do usuário.

\subsubsection{Procedimentos para Desenvolver o Sistema RFID}

O Raspberry PI Zero W foi utilizado como microcontrolador para habilitar o leitor MFRC-522 do sistema RFID a se comunicar via protocolo MQTT com o gateway, para o qual são enviados os dados coletados. Para tanto, o Raspberry PI Zero W se conecta à rede Wi-Fi local do Raspberry PI 3 (gateway). A linguagem de programação utilizada nesta etapa foi Python3. O procedimento inicial para permitir a aplicação desenvolvida consistiu em quatro etapas: (1) instalação do sistema operacional Raspbian; (2) instalação dos pacotes necessários para a aplicação em Python; (3) configuração do Raspberry PI Zero W para acessar automaticamente a rede local do gateway e (4) instalação dos componentes físicos do circuito elétrico. 


\subsubsection{Procedimentos para Desenvolver da Interface de Visualização BIM}

O software Unity foi utilizado como plataforma de desenvolvimento dos recursos visuais, servindo como suporte para os modelos BIM e possibilitando a comunicação com a web por meio do WebGL. Os procedimentos necessários para estabelecer esta comunicação consistiram nas seguintes etapas: (1) importação do modelo BIM para o Unity; (2) ajuste de funcionalidades de visualização do modelo; (3) estabelecimento de comunicação entre o modelo e o banco de dados e (4) exportação do modelo em WebGL.

\section{Resultados}

Como resultado obteve-se o gateway desenvolvido com capacidade de: 1) gerenciar a coleta dos dados; 2) selecionar e processar os dados relevantes; 3) armazenar localmente os dados e 4) servir de interface para agregar informações de interesse no banco de dados para posterior alimentação do modelo BIM, onde as informações são contextualizadas.

\subsection{Sistema RFID}

Conforme Figura 3, para representar e habilitar a leitura e/ou cadastro de uma nova tag no sistema, 1 botão (push button) foi inserido no circuito, o qual deve ser pressionado pelo usuário antes da leitura da tag de interesse. Para representar alguns possíveis status representativos dos recursos físicos que poderiam ser capturados na obra através do sistema RFID, mais 3 botões (push buttons) foram inseridos no sistema. O primeiro botão representa o status produção; o segundo o status transporte e o terceiro o status montagem do recurso físico. Antes de cada leitura da tag o usuário deve pressionar um dos botões, conforme correspondência com a situação real, para que o dado coletado seja registrado e enviado de forma correta ao banco de dados. Os tipos de dados registrados no banco a partir das leituras são: UID da tag lida, data, horário e status.

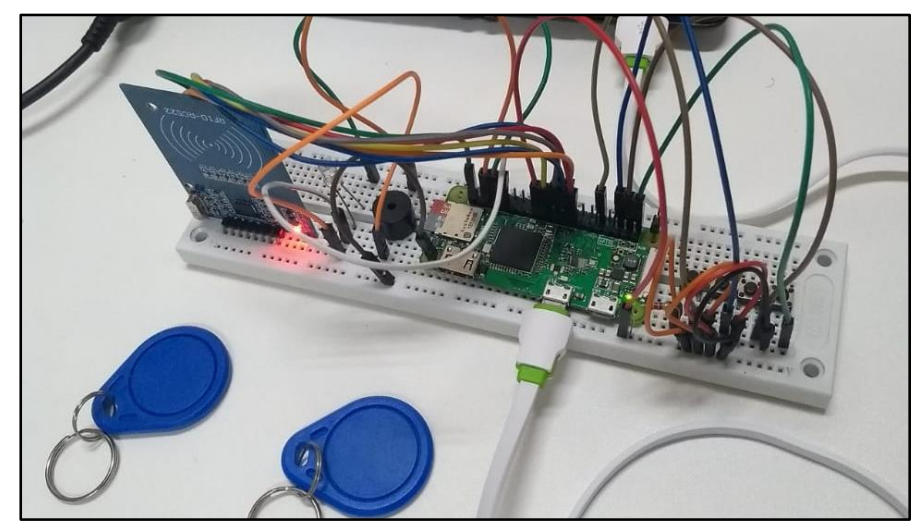

Figura 3: Funcionamento do sistema RFID.

Antes de cada leitura o LED acende indicando que a leitura deve ser realizada. Uma vez que a tag tenha sido lida, o sistema RFID faz o envio dos dados via MQTT diretamente para o broker. Ao final desta operação, o buzzer emite um sinal sonoro e o LED se apaga. Foi configurado um intertravamento entre os quatro botões para garantir que só uma operação seja realizada por vez. 


\subsection{Gateway}

Conforme indicado na Figura 4, o gateway foi desenvolvido a partir do dispositivo Raspberry PI 3, que atua como servidor local. Para lidar com os dados trocados através da IoT foi utilizado o Eclipse Mosquitto, que é um broker MQTT de código aberto. A linguagem de programação utilizada em todo este processo foi Python2. Para permitir sua comunicação com sistema RFID, o gateway foi habilitado para gerar a rede Wi-Fi local própria.

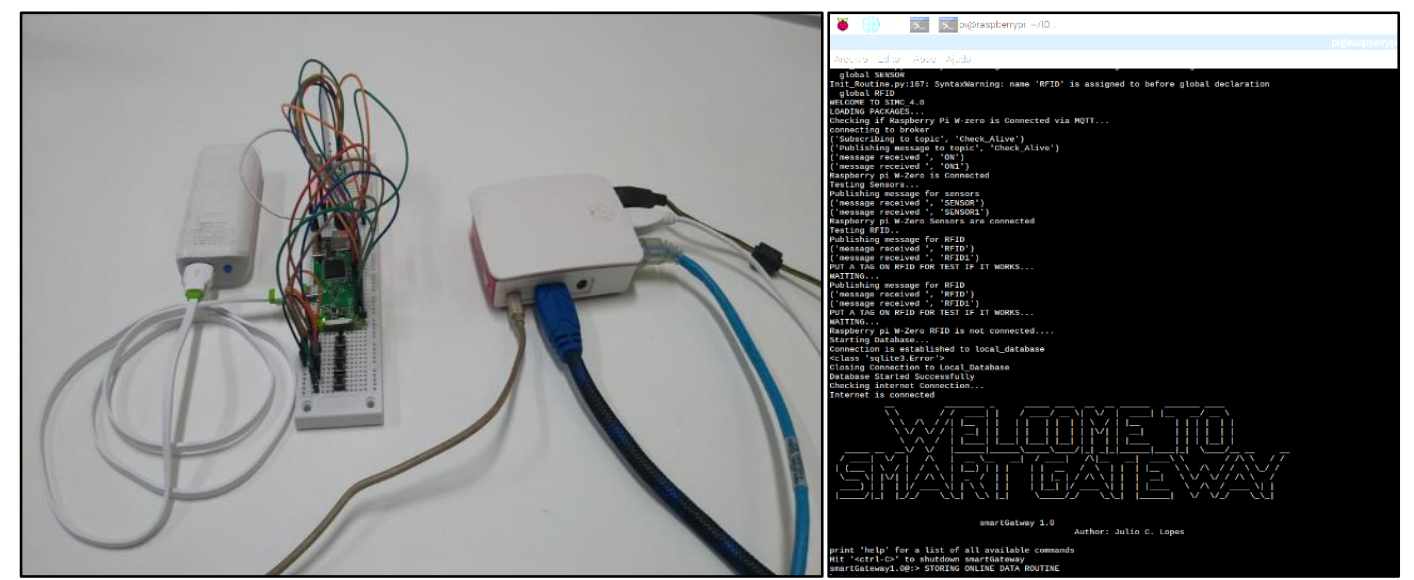

Figura 4: Funcionamento do Gateway através do Raspberry Pi 3.

Como no canteiro de obras nem sempre está disponível uma conexão com uma rede externa sem fio, o gateway é capaz de funcionar offline armazenando os dados em um banco de dados SQL local através da biblioteca SQLite3. Todos os dados coletados com o sistema RFID ficam armazenados localmente antes de serem enviados ao banco de dados em nuvem, conforme Figura 5. Caso haja a conexão externa, os dados serão enviados diferetamente para a nuvem após serem coletados pelo gateway.

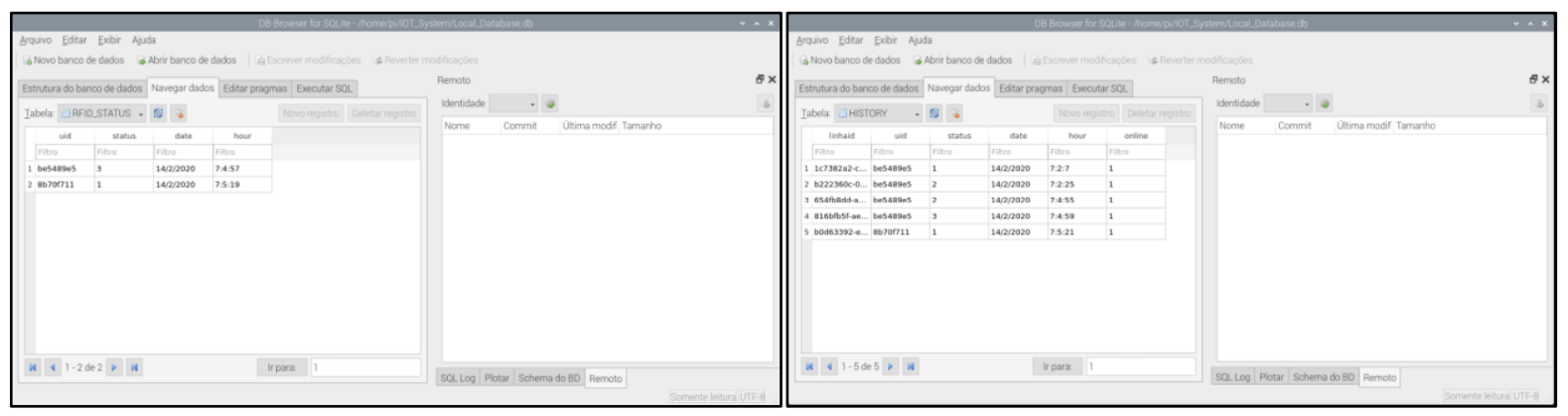

Figura 5: Banco de dados local do Raspberry Pi 3.

A inicialização do gateway consiste em algumas etapas: (1) automaticamente ele realizará uma verificação completa de todas as funções do sistema para garantir que a inicialização foi feita com sucesso. Caso haja algum erro de comunicação ou falha de inicialização, o próprio sistema detectará o erro e comunicará ao usuário para que seja feito o ajuste; (2) uma vez inicializado, o sistema contará com três loops para o seu funcionamento. O primeiro loop é o MQTT, que estará recebendo as mensagens MQTT e realizando funções de acordo com o conteúdo das mensagens recebidas; o segundo loop é o que irá verificar se existe conectividade com a rede 
externa em um intervalo definido pelo usuário e o terceiro loop é o Menu, que se trata de uma interface que pode ser acessada diretamente pelo usuário e que possibilita a realização de funções com o sistema RFID remotamente por meio de protocolo MQTT, além de acesso ao banco de dados local e ao online.

O gateway foi configurado para enviar os dados armazeados localmente para o Firebase quando verificar a conexão com redes externas de internet. Neste experimento, tal rede consiste em uma WLAN cabeada. Os dados no Firebase possuem a estruturação indicada na Figura 6:

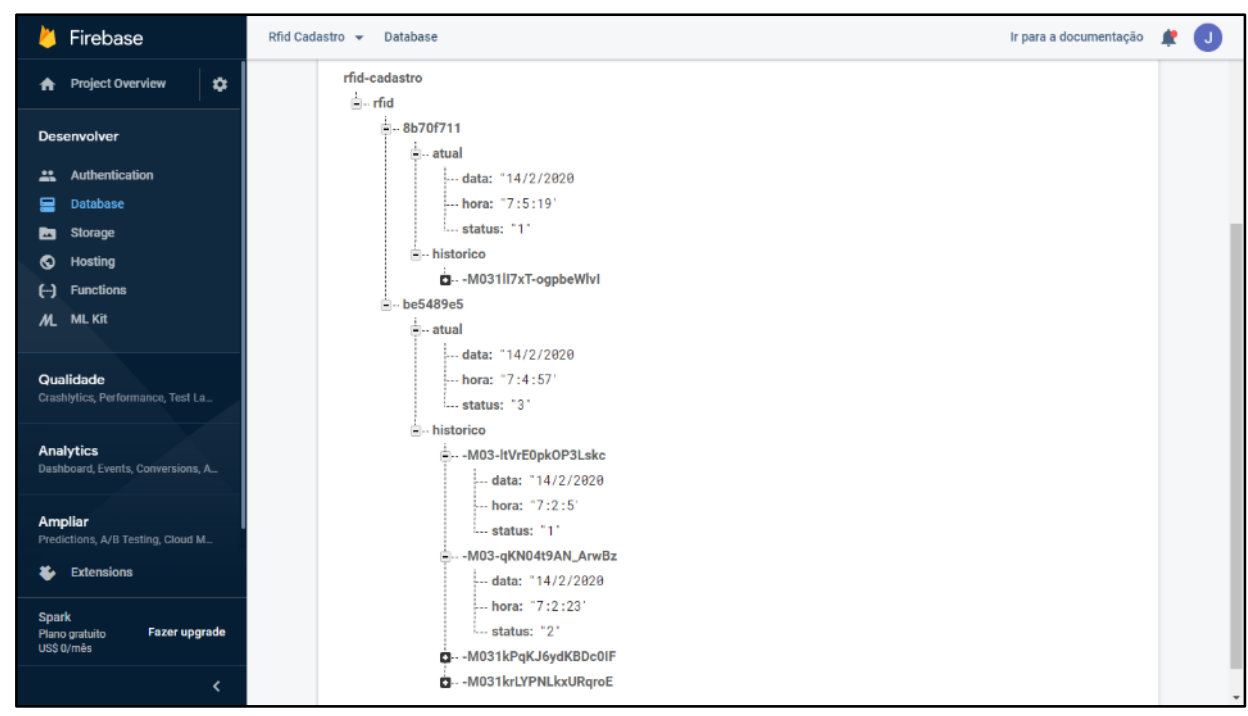

Figura 6: Funcionamento do banco de dados no Firebase.

\subsection{BIM}

Conforme a Figura 7, foi possivel estabelecer a comunicação do banco de dados com as possíveis áreas de utilização do BIM. Esta aplicação foi desenvolvida utilizando o software Unity, tendo como objetivo gerar uma visualização em WebGL. O modelo BIM desenvolvido pelo software Revit foi exportado no formato $f b x$ e posteriormente importado para o Unity. Em seguida, foi necessário estabelecer funções de apoio a visualização do modelo, como a rotação central. Posteriormente foi estabelecido a comunicação entre o modelo BIM e o Banco de Dados Firebase atraves do REST API, que possibilitou a comunicação entre o WebGL e o banco de dados instantaneamente. Esta comunicação consistiu no processo de enviar e receber dados para o banco de dados, utilizando artifícios como botões virtuais que desencadearam à ação de leitura do status atual do elemento desejado por meio da interface WebGL. Por fim, foi realizado a exportação do modelo para a versão WebGL e utilizada no site $<$ https://br.000webhost.com/> como hospedagem teste, conforme Figura 8. Este resultado mostra que o processo de comunicação e integração entre o BIM e a IoT foi realizado com êxito e que diferentes aplicações podem ser estabelecidas através deste processo.

Como objetivos futuros, os autores estão desenvolvendo aplicações práticas em indústrias de pré-fabricados, utilizando o gateway desenvolvido para dar suporte à interface de comunicação por meio de BIM, IoT e RFID, visando auxiliar no gerenciamento dos processos de inspeção de qualidade e, principalmente, no acompanhamento logístico dos elementos pré-fabricados. Além disso, estão sendo avaliadas aplicações para outros recursos físicos, em especial as formas metálicas utilizadas no sistema construtivo de paredes de concreto. 


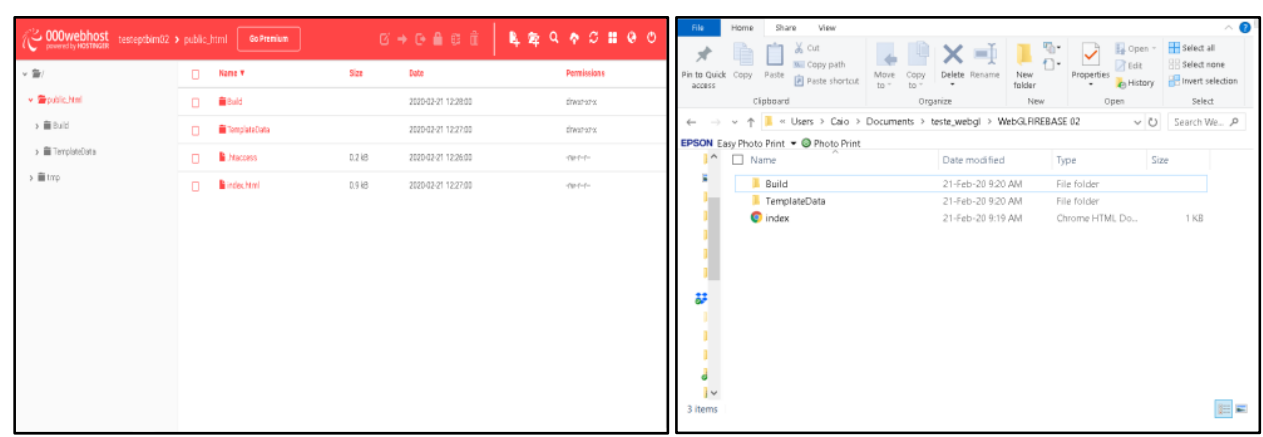

Figura 7: Arquivos WebGL gerados por meio do Unity e exportados para o site.

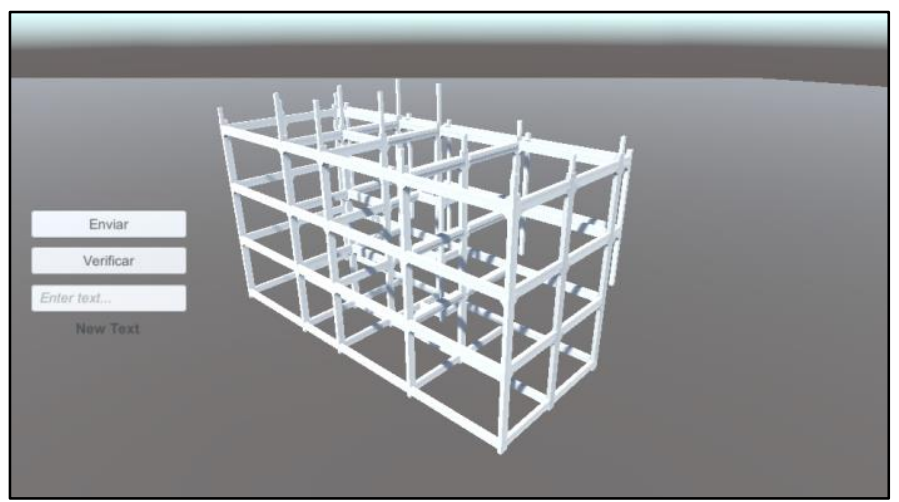

Figura 8: Representação do modelo BIM através da web.

\section{Considerações Finais}

As principais aplicações previstas para gateway são nas áreas de rastreamento de recursos físicos e de processos, planejamento e controle de produção e controle de qualidade. Exemplos de recursos físicos que podem ser rastreados são elementos pré-fabricados ou formas metálicas utilizadas em processos de concretagem. O objetivo final da aplicação é que, a partir do rastreamento, o modelo BIM funcione como um gêmeo digital da obra, registrando e exibindo em tempo real status correspondentes, por exemplo, com a produção, transporte e montagem ou qualidade destes recursos no campo. O experimento realizado apresentou resultados satisfatório considerando o contexto da construção civil, demonstrando a sua relevância e necessidade em uma aplicação de IoT. Apesar dos testes terem sido realizados de forma simplificada foi possível observar a comunicação entre os elementos envolvidos, exibindo a possibilidade de aplicação do mesmo sistema em outras circunstâncias. A principal limitação encontrada se refere à atual necessidade de uma rede externa cabeada, que deverá futuramente ser substituída por uma rede sem fio. As aplicações em campo serão indicadas em publicações futuras, onde será possível analisar os desafios encontrados em um ambiente mais complexo.

\section{Referências}

[1] L. Andrade, C. Lira, B. Mello, A. Andrade, A. Coutinho, F. Greve, and C. Prazeres," Do device à cloud com a plataforma SOFT-IoT: sua infraestrutura IoT em poucas horas," Anais do XXIV Simpósio Brasileiro de Sistemas Multimídia e Web: Minicursos, 2018. 
[2] L. Atzori, A. Iera, and G. Morabito, "The Internet of Things: a survey," in Computer Networks (2010), vol. 54, n. 15, pp. 2787-2805, 2010.

[3] K. Chen, W. Lu, Y. Peng, S. Rowlinson, and G. Q. Huang, "Bridging BIM and building: from a literature review to an integrated conceptual framework,". in Int J. Proj Manage, vol. 33, pp.1405-1416, 2015.

[4] K. Chen, W. Lu, F. Xue, L. Zheng, D. Liu, "Smart gateway for bridging BIM and building," in Proceedings of the 21st International Symposium on Advancement of Construction Management and Real Estate (2018), Singapore, 2018.

[5] C. M. Eastman, P. Teicholz, R. Sacks, and K. Liston, BIM handbook: a guide to building information modeling for owners, managers, designers, engineers, and contractors. 2. ed. Hoboken, N.J.: Wiley, 2011.

[6] X. Gao, and S. Tang, P. Pishdad-bozorgi, and D. Shelden, "Foundational Research in Integrated Building Internet of Things (IoT) Data Standards," Center for the Development and Application of Internet of Things Technologies, Georgia Institute of Technology, 2018.

[7] A. C. Gil, "Como Elaborar Projetos de Pesquisa," 4. ed. São Paulo: Atlas, 2002.

[8] U. Isikdag, "Enhanced Building Information Models," 1.ed. Istanbul: Springer, 2015.

[9] C. Z. Li, F. Xue, X. Li, and J. Hong, SHEN, G. Q. "An Internet of Things-enabled BIM platform for on-site assembly services in prefabricated construction," Automation in Construction, vol.89, pp. 146-161, 2018.

[10] M. A. Marconi, and E. M. Lakatos, "Técnicas de Pesquisa” 7. ed. São Paulo: Atlas, 2008.

[11]T. D. Oesterreich, and F. Teuteberg, "Understanding the implications of digitisation and automation in the context of Industry 4.0: A triangulation approach and elements of a research agenda for the construction industry," Computers in Industry, vol.83, p.121-139, 2016.

[12] J. Park, Y. Cho, C. Ahn, "A Wireless Tracking System Integrated with BIM for Indoor Construction Applications," In: Construction Research Congress (2016), San Juan. Proceedings... San Juan: 2016, p. 2660-2668.

[13] V. Roblek, M. Meško, and A. Krapež, "A Complex View of Industry 4.0," SAGE Open, pp. 1-11, 2016. doi:10.1177/2158244016653987.

[14] H. Sundmaeker, P. Guillemin, P. Friess, and S. Woelfflé. "Vision and challenges for realising the Internet of Things, Cluster of European Research Projects on the Internet of Things," 1.ed. Luxembourg: Publications Office of the European Union, 2010.

[15]L. Thames and D. D. Schaefer, "Software-defined Cloud Manufacturing for Industry 4.0," Changeable, Agile, Reconfigurable \& Virtual Production Conference (2016), Bath, UK, 2016, vol. 52, pp. 12-17. doi: 10.1016/j.procir.2016.07.041

[16]R. Want. "An introduction to RDIF technology,"IEEE Pervasive Computing (2006), Urumqi, China, vol. 5, n. 1, pp. 25-33. doi: 10.1109/MPRV.2006.2

[17] Y. Zhai, K. Chen, J. X. Zhou, J. Cao, Z. Lyu, X. Jin, G. Q. P. Shen, W. Lu, and G. Q. Huang, "An Internet of Things-enabled BIM platform for modular integrated construction: A case study in Hong Kong," Advanced Engineering Informatics, vol. 42, pp. 1-15, April 2019.

[18] R. Y. Zhong, Y.Peng, F. Xue, J. Fang, W. Zou, H. Luo, S. T. Ng, W. Lu, G. Q. P. Shen, G. Q. Huang, "Prefabricated construction enabled by the Internet-of-Things," Automation in Construction. vol.76, pp. 59-70, December 2017. 